\title{
Characterization of bidirectional gene pairs in The Cancer Genome Atlas (TCGA) dataset
}

\author{
Juchuanli Tu ${ }^{1,2}$, Xiaolu Li ${ }^{2}$, Jianjun Wang ${ }^{\text {Corresp. } 3}$ \\ ${ }^{1}$ College of Life Sciences, Beijing Normal University, Beijing, China \\ 2 National Institute of Biological Sciences, Beijing, China \\ ${ }^{3}$ State Key Laboratory of Microbial Resources, Institute of Microbiology, Chinese Academy of Sciences, beijing, China \\ Corresponding Author: Jianjun Wang \\ Email address: wangjj@im.ac.cn
}

The "bidirectional gene pair" indicates a particular head-to-head gene organization in which transcription start sites of two genes are located on opposite strands of genomic DNA within a region of $1 \mathrm{~kb}$. Despite bidirectional gene pairs are well characterized, little is known about their expression profiles and regulation features in tumorigenesis. We used RNA-seq data from The Cancer Genome Atlas (TCGA) dataset for a systematic analysis of the expression profiles of bidirectional gene pairs in 13 cancer datasets. Gene pairs on the opposite strand with transcription end site distance within $1 \mathrm{~kb}$ or on the same strand with the distance of two genes between $1 \mathrm{~kb}-10 \mathrm{~kb}$ and gene pairs comprising two randomly chosen genes were used as control gene pairs (CG1, CG2 and random). We identified and characterized up-/down-regulated genes by comparing expression level between tumors and adjacent normal tissues in 13 TCGA datasets. There were no consistently significant difference in percentage of up-/down-regulated genes between bidirectional and control/random genes in most of TCGA datasets. However, the percentage of bidirectional gene pairs comprising two up- or two down-regulated genes was significantly higher than gene pairs from CG1/2 in 12/11 analyzed TCGA datasets and the random gene pairs in all 13 TCGA datasets. Then we identified the methylation correlated bidirectional genes to explore the regulatory mechanism of bidirectional genes. Like the differentially expressed gene pairs, the bidirectional genes in a pair were significantly prone to be both hypo- or hyper-methylation correlated genes in 12/13 TCGA datasets when comparing to the CG2/random gene pairs despite of no significantly difference between the percentages of hypo-/hyper-methylation correlated genes in bidirectional and CG2/random genes in most of TCGA datasets. Finally, we explored the correlation between bidirectional genes and patient's survival, identifying prognostic bidirectional genes and prognostic bidirectional gene pairs in each TCGA dataset. Remarkably, we found a group of prognostic bidirectional gene pairs in which combination of two protein coding genes with different expression 
level correlated with different survival prognosis in survival analysis for OS. The percentage of these gene pairs in bidirectional gene pair were significantly higher than the gene pairs in controls in COAD datasets and lower in none of 13 TCGA datasets. 
1 Characterization of bidirectional gene pairs in The Cancer Genome Atlas (TCGA)

2 dataset

3 Juchuanli $\mathrm{Tu}^{1}, 2$, Xiaolu $\mathrm{Li}^{2}$ and Jianjun Wang ${ }^{3^{*}}$

$4{ }^{1}$ College of Life Sciences, Beijing Normal University, Beijing, China

$5 \quad 2$ National Institute of Biological Sciences, Beijing, China

$6{ }^{3}$ State Key Laboratory of Microbial Resources, Institute of Microbiology, Chinese

7 Academy of Sciences, Beijing, China

9 Address correspondence to: Jianjun Wang, State Key Laboratory of Microbial Resources, Institute of Microbiology, Chinese Academy of Sciences, Beijing, China, NO.1

11 Beichen West Road, Chaoyang District, Beijing 100101, China. Fax: +86-10-64807429,

12 Tel: +86-10-64807417, Email address: wangjj@im.ac.cn

13

14

15

16

17

18

19

20

Running Title: Characterization of bidirectional gene pairs in The Cancer Genome Atlas (TCGA) dataset 


\section{Abstract}

31 The "bidirectional gene pair" indicates a particular head-to-head gene organization in which transcription start sites of two genes are located on opposite strands of genomic DNA within a region of $1 \mathrm{~kb}$. Despite bidirectional gene pairs are well characterized, little is known about their expression profiles and regulation features in tumorigenesis. We 
54

prognostic bidirectional genes and prognostic bidirectional gene pairs in each TCGA dataset. Remarkably, we found a group of prognostic bidirectional gene pairs in which combination of two protein coding genes with different expression level correlated with different survival prognosis in survival analysis for OS. The percentage of these gene pairs in bidirectional gene pair were significantly higher than the gene pairs in controls in COAD datasets and lower in none of 13 TCGA datasets.

Keywords: bidirectional gene pair, differentially expressed gene, DNA methylation, survival analysis

\section{Introduction}

The "bidirectional gene pair" indicates a particular "head-to-head" gene organization in which transcription start sites (TSSs) of two genes are located on opposite strands of genomic DNA within a region of $1 \mathrm{~kb}$ (Lin et al. 2007; Liu et al. 2011; Trinklein et al. 2004; Yang et al. 2007). The intervening regulatory region is called "bidirectional promoter" (Liu et al. 2011). Bidirectional genes have been thought to represent more than $10 \%$ of human genes (Yang et al. 2007). The architecture of bidirectional gene pairs is evolutionally conserved, thus suggesting a functional importance (Koyanagi et al. 2005; Trinklein et al. 2004; Yang \& Elnitski 2014). Indeed, some of bidirectional genes are often associated with functions in DNA repair, with the potential to participate in the development of cancer (Adachi \& Lieber 2002; Koyanagi et al. 2005; Yang et al. 2007). Previous studies indicated that bidirectional gene pairs are associated with several genomic regulatory features such as $\mathrm{CpG}$ island methylation and histone modification (Adachi \& Lieber 2002; Jangid et al. 2018; Lin et al. 2007; Shu et al. 2006; Trinklein et al. 2004).

As previously indicated, bidirectional gene pairs are related to crucial cellular functions and contribute to tumorigenesis (Adachi \& Lieber 2002; Yang et al. 2007). However, the majority of these studies are based on genomic features without considering the real 
80

81

expression level of bidirectional gene pairs in cancer. Besides, little is known about the regulation mechanisms underlying bidirectional gene pairs involved in tumorigenesis. Here, we used RNA-seq data from TCGA dataset for a systematic analysis of the expression profiles of bidirectional genes and gene pairs in cancer (Cancer Genome Atlas 2012a; Cancer Genome Atlas 2012b; Cancer Genome Atlas Research 2012; Cancer Genome Atlas Research 2014a; Cancer Genome Atlas Research 2014b; Cancer Genome Atlas Research et al. 2013). We identified and characterized the up/down-regulated bidirectional genes and gene pairs in 13 TCGA datasets. We also identified and characterized the methylation correlated bidirectional genes to explore the regulatory mechanism of bidirectional genes. Besides, we evaluated the prognosis of bidirectional genes by survival analysis to correlate the expression of bidirectional genes with clinical outcome.

\section{Materials \& Methods}

\section{Identification of bidirectional, control and random gene pairs in the human} genome

The gene annotation GDC.h38 v.22 was retrieved from GENCODE (https://gdc.cancer.gov/about-data/data-harmonization-and-generation/gdc-reference-

files). The definition of gene type for each gene was included in the annotation file. For identifying bidirectional gene pairs, we used a method proposed in a previous work (Trinklein et al. 2004). Briefly, for each gene we defined the position of the transcription start site (TSS) and the transcription end site (TES) as the 5'-most boundary and the 3'most boundary transcripts of the analyzed gene, respectively (head-to-head structure). We defined bidirectional genes as two genes on opposite strand with TSS distance within $1 \mathrm{~kb}$. We excluded that gene pairs in which a gene was entirely located in another gene, a condition commonly defined as nested genes. 
105 For identifying two control gene pairs (CG1 and CG2), we followed the same way

106

107

108

109

110

111

112

113

114

115

116

117

118

119

120

121

122

123

124

125

126

127

128

129

130

except for searching the gene pairs on the opposite strand with TES distance within 1

$\mathrm{kb}$ as control gene pairs 1 (CG1) and on the same strand with the distance of two genes between $1 \mathrm{~kb}$ and $10 \mathrm{~kb}$ as control gene pairs 2 (CG2) (Trinklein et al. 2004). Finally we excluded control gene pairs which contain bidirectional gene.

Since the number and the composition of gene types for bidirectional gene pairs and control gene pairs above was difference, we also collected a set of gene pairs comprising two genes randomly chosen in the genome as control. The number and the composition of gene types for each set of random gene pairs was the same as bidirectional gene pairs. We repeated 100 times of selections and got 100 sets of random gene pairs.

\section{TCGA expression datasets and analysis}

All TCGA expression datasets from RNA-seq were downloaded from the TCGA website (https://portal.gdc.cancer.gov/). Raw reads counts were extracted from files with the suffix "htseq.counts". We only incorporated cancer datasets with $\geq 200$ cancer samples and $\geq 15$ normal samples for an accurate identification of differentially expressed genes. The normal samples were defined as the samples marked as "Solid Tissue Normal" and no blood samples were included into the analysis as normal samples. A total of 13 cancer datasets matched these criteria. All subsequent analyses were performed on these 13 cancer datasets except for the methylation data analysis. We excluded the STAD dataset in methylation analysis for the methylation data available for only two normal samples in this dataset.

We employed the "RUVg" function in "RUVSeq" package (v3.8) to correct the batch effect in RNA-seq datasets following the previous studies (Aran et al. 2017; Risso et al. 2014). The identification of differentially expressed genes was performed by "glmTreat" function in "edgeR" package (v3.24.0) (Robinson et al. 2010). We kept genes in each 
131 TCGA dataset when their count-per-million (CPM) was $\geq 0.1$ in at least the size of

132 normal samples and defined these genes as expressed genes. Then we identified the

133 differentially expressed genes by comparing the expression profile of a specific gene

134 between cancer and normal sample groups. Genes with FDR $\leq 0.05$ and expression

135 fold change $\geq 1.5$ (up-regulated genes) or $\leq 0.67$ (down-regulated genes) were defined

136 as differentially expressed genes (Xiao et al. 2018). The trimmed mean of M-values

137 (TMM) normalized expression value was also generated by "edgeR" package.

138

139

140

141

142

143

144

145

146

147

148

149

150

151

152

153

154

155

\section{Gene ontology (GO) analysis}

The gene ontology (GO) analysis was performed on the DAVID GO bioinformatics platform (V6.8) (https://david.ncifcrf.gov/) (Dennis et al. 2003). The ensembl gene ID for each gene was used as the input. Genes which were not recognized by DAVID GO website were excluded from GO analysis.

\section{Correlation analysis of methylation level and bidirectional genes and gene pairs}

The DNA methylation data from Human Methylation 450 BeadChip platform were downloaded from TCGA website (https://portal.gdc.cancer.gov/). We extracted betavalues to evaluate the DNA methylation level of each probe. The annotations of probes to specific genes were defined as these probes were located on the promoter region of genes. The promoter region was defined as the region from $2 \mathrm{~kb}$ upstream to $500 \mathrm{bp}$ downstream of TSS of a gene (Tang \& Epstein 2007). We excluded the STAD dataset for the methylation data available for only two normal samples.

We used the "champ.DMP" function in the "ChAMP" package in R to identify differentially methylation probes (Morris et al. 2014). We defined probes with adjusted pvalue $\leq 0.05$ as differentially methylation probes.

Then we defined a gene as correlated to methylation when this gene was up-regulated in cancer with at least one hypo-methylated probe was annotated to this gene, or when 
156

157

158

159

160

161

162

163

164

165

166

167

168

169

170

171

172

173

174

175

176

177

178

179

180

181

this gene was down-regulated in cancer with at least one hyper-methylated probe was annotated to this gene.

\section{Survival analysis in TCGA datasets}

The survival analysis was basically performed as previously reported (Anaya et al. 2015). Briefly, for each cancer dataset, survival information were retrieved from the "TCGA-CDR" dataset which keep for each patient the most recent follow up information (Liu et al. 2018). Since the information of disease-specific survival (DSS) is approximated in most of TCGA datasets, we excluded it from our analysis and kept other three endpoints (Overall Survival (OS), disease-free interval (DFI) and progression-free interval (PFI)).

Cox models were run with the "coxph" function from the "survival" package in $R$, and the equation for the bidirectional genes is "coxph(Surv(time,censor) exprs)", where time is survival time for OS or disease/ progression-free interval time for DFI/PFI, censor is survival even for OS or interval event for DFI/PFI for each patient, and exprs is the TMM normalized expression value for each gene.

For the bidirectional gene pairs, the equation is " $\operatorname{coxph}(\operatorname{Surv}($ time,censor) exprs1+exprs2)", where exprs1 and exprs2 are the TMM normalized expression value for gene1 and gene2 in a pair. We marked genes or gene pairs with Logrank p-value $\leq 0.05$ as prognostic genes or gene pairs.

For every prognostic bidirectional gene pair, we divided the samples into four groups based on the combination of expression level of two genes in a pair (high_vs_high, low_vs_low, high_vs_low and low_vs_high). The cutoff to classify the groups was the median value of TMM normalized expression value for gene1 and gene2 in a pair. Then Cox models were run with the "coxph" function, and the equation for the bidirectional gene pairs is "coxph(Surv(time,censor) group)". We picked the bidirectional gene pairs with Logrank $p$-value $\leq 0.05$ and considered that these bidirectional gene pairs as the 
182

183

184

185

186

187

188

189

190

191

192

193

194

195

196

197

198

199

200

201

202

203

204

205

206

pairs in which the combination of two genes with different expression level correlated with different survival outcome.

We did not performed multiple testing adjustment for survival analysis, since previous study showed that the number of prognostic genes was no more than 50 in 9 out of 16 TCGA datasets and even 0 in 5 out of 16 TCGA datasets after adjustment (Anaya et al. 2015).

\section{Results}

\section{Identification of bidirectional gene pairs in the human genome}

We used the GENCODE gene annotation (v22) for identifying the bidirectional gene pairs present in the human genome. According to previous analyses, we defined two genes located on the opposite strands, whose transcription start sites (TSSs) were separated by less than $1 \mathrm{~kb}$ pairs, as bidirectional gene pairs (BG, head-to-head structure) (Trinklein et al. 2004). Meanwhile, we identified two groups of control gene pairs, which were named as control gene pairs 1 and 2 (CG1 and CG2) (Fig. 1A). Overall, we identified 4083 bidirectional gene pairs (BG), 1054 control gene pairs 1 (CG1) and 13037 control gene pairs 2 (CG2). The number of bidirectional genes accounted for $12.9 \%$ of all human genes, which was consistent with previous study (Yang et al. 2007). Detailed information about all bidirectional and control gene pairs are listed in the Supplemental file 1.

Among the identified bidirectional genes, $57.4 \%$ were protein coding genes. The top four gene types of bidirectional genes accounted for $89.2 \%$ of all bidirectional genes (Fig. 1B). The top four combination of gene types accounted for $75.3 \%$ of bidirectional gene pairs (Fig. 1C).

We noticed that the number and the composition of gene types for bidirectional gene pairs were different from the two control gene pairs. In order to rule out the possible bias 
207

208

209

210

211

212

213

214

215

216

217

218

219

220

221

222

223

224

225

226

227

228

229

230

231

232

derived from these difference, we introduced an additional control gene pairs comprising two genes randomly chosen from genome which were named as random gene pairs (Fig. 1A). The number and the composition of gene types for random gene pairs kept the same as bidirectional gene pairs (Fig. S1A and S1B). We repeated 100 times to get 100 sets of random gene pairs to avoid sampling bias.

The gene function enrichment analysis showed that terms such as DNA repair, DNA replication and replication fork processing were enriched in bidirectional genes, accordingly with previous study (Fig. 1D) (Adachi \& Lieber 2002).

\section{Identification and characterization of up-/down-regulated bidirectional genes in} TCGA dataset

Previous studies showed that abnormal expressed genes play a crucial role in tumorigenesis and bidirectional genes may participate in the development of cancer (Adachi \& Lieber 2002; Hanahan \& Weinberg 2011; Lee \& Young 2013; Yang et al. 2007). However, few study systematically explored and characterized the expression profile of bidirectional genes and gene pairs in cancer. Hence, we set out to identify the differentially expressed bidirectional genes and gene pairs in 13 TCGA datasets.

Detailed information of all the 13 analyzed TCGA datasets was shown in Supplemental Table 1.

We used the "RUVSeq" package to correct the batch effect (Aran et al. 2017; Risso et al. 2014) and then used the "edgeR" package to identify the differentially expressed genes (Robinson et al. 2010). The method we followed involved first removing genes with very low expression which we defined as non-expressed genes. After filtration, about $50 \%$ of the all human genes in each TCGA dataset were defined as nonexpressed and were removed from the subsequent analysis (Fig. S1C and Supplemental file 2). Surprisingly, only about $20 \%$ bidirectional genes in each TCGA dataset were identified as non-expressed genes (Fig. 2A). Meanwhile $\sim 30 \%$ control and 
233

234

235

236

237

238

239

240

241

242

243

244

245

246

247

248

249

250

251

252

253

254

255

256

257

258

random genes were identified as non-expressed genes (Fig. 2B, S1D and S1E). The difference between the percentage of expressed genes in bidirectional genes and control/random genes in each of 13 TCGA datasets was significant ( $p$-value $\leq 0.001$, "chisq.test" in R). The results indicated that bidirectional genes were prone to be expressed when comparing with control and random genes.

We then identified differentially expressed bidirectional genes by comparing the expression profiles between groups of cancer and normal samples in the 13 TCGA datasets (Supplemental file 3). Although we witnessed significantly different percentage of up-/down-regulated genes between bidirectional genes and control genes in some TCGA datasets. In most of TCGA datasets there were no significant difference in the percentage of up- or down-regulated genes between bidirectional genes and control genes (Fig. 2C, 2D, S2A and S2B). We witnessed similar results by comparing percentage of up-/down-regulated genes in bidirectional genes and random genes (Fig. S3A and S3B). These results indicated that there were no consistently significant difference in percentage of up-/down-regulated genes between bidirectional and control/random genes in most of TCGA datasets.

\section{Identification and characterization of up-/down-regulated bidirectional gene pairs} in TCGA dataset

Next, we evaluated the combination of expressed and non-expressed bidirectional genes in a pair in each TCGA dataset. Our results showed that more than $65 \%$ bidirectional genes in one pair were both expressed. Meanwhile no more than $60 \%$ control and random genes in a pair were both expressed (Fig. 3A, 3B, S2D and S3D). The difference between the percentages of expressed gene pairs from bidirectional and control/random gene pairs were significant in all 13 TCGA datasets ( $p$-value $\leq 0.001$, "chisq.test" in R). The results indicated that bidirectional genes in a pair were prone to be both expressed when comparing with control and random gene pairs. 
259

260

261

262

263

264

265

266

267

268

269

270

271

272

273

274

275

276

277

278

279

280

281

282

283

284

Next, we explored the distribution of combination of up-/down-regulated bidirectional and control genes in one pair. Although the percentage of bidirectional gene pairs comprising two up- or two down-regulated genes were not always higher than the control gene pairs, we witnessed that the percentage of bidirectional gene pairs comprising two up-regulated genes plus bidirectional gene pairs comprising two downregulated genes were significantly higher than CG1 except for LUSC dataset and CG2 except for KIRP and UCEC datasets (Fig. 3C, 3D and S2C, p-value $\leq 0.05$, "chisq.test" in $\mathrm{R}$ ). We also checked the difference between the distribution of combination of up/down-regulated bidirectional and random genes in one pair. It showed that the percentage of bidirectional gene pairs comprising two up-regulated genes plus bidirectional gene pairs comprising two down-regulated genes were significantly higher than the same percentage of combination in random gene pairs in all analyzed TCGA datasets (Fig. 3C and S3C, p-value $\leq 0.001$, "chisq.test" in R).

\section{Characterization of up-/down-regulated bidirectional genes and gene pairs in} multiple TCGA datasets

Next, we explored up-/down-regulated bidirectional genes and gene pairs in multiple TCGA datasets. If a bidirectional gene or gene pair is up- or down-regulated in multiple cancer datasets, it indicates a common regulation and function across multiple cancer types. On the other hand, it may suggest a cancer-type specific regulation and function. We summarized the frequency of bidirectional, control and random genes up-/downregulated in a specific number of TCGA datasets (Fig. 4A, 4B and Supplemental file 4). Although distribution of up-regulated bidirectional genes among multiple TCGA datasets was significantly skewed to the right when comparing to the CG2 and random genes (Fig. 4A) and distribution of down-regulated bidirectional genes among multiple TCGA datasets was also significantly skewed to the right when comparing to the CG2 (Fig. 4B). The distribution of up-/down-regulated bidirectional genes showed no consistently 
285

286

287

288

289

290

291

292

293

294

295

296

297

298

299

300

301

302

303

304

305

306

307

308

309

310

significant pattern when comparing with the distribution of control/random genes.

Then we evaluated the distribution of bidirectional gene pairs comprising specific combination of up-/down-regulated genes among all the 13 analyzed TCGA datasets.

We observed that the distribution of bidirectional gene pairs comprising two up- or two down-regulated genes was significantly skewed to the right when comparing to the control and random gene pairs (Fig. 4C and Supplemental file 5). On the other hand, we also explored the distribution of gene pairs comprising one up- and one down-regulated genes. The result showed that the distribution of specific bidirectional gene pairs was significantly skewed to the left when comparing with CG1 or random gene pairs and no significantly different from CG2 (Fig. 4D).

Together, the results showed that the bidirectional gene pairs were prone to be up- or down-regulated in multiple TCGA datasets despite of the distribution of up- or downregulated bidirectional genes showing no consistently significant difference from control or random genes.

\section{Correlation between DNA methylation and bidirectional genes}

Previous study reported that $\mathrm{CpG}$ islands were enriched in the promoter region of bidirectional gene pairs (Adachi \& Lieber 2002). A recent study also witnessed the change in methylation was significantly greater in control compared to bidirectional promoters in cancer (Thompson et al. 2018). However, no systematical analyses have been performed for identifying and characterizing the methylation level correlated to bidirectional genes in cancer.

Here, we performed the pan-cancer analysis of methylation correlated to bidirectional genes. We defined that a methylation probe was hypo-methylated when the beta-value of this probe in cancer group was significantly lower than in normal group, while the hyper-methylated probe when the beta-value in cancer group was significantly higher than in normal group (Morris et al. 2014). The detailed information of hypo-/hyper- 
311 methylated probes can be viewed in Supplemental file 6. Then we defined a gene as

312

313

314

315

316

317

318

319

320

321

322

323

324

325

326

327

328

329

330

331

332

333

334

335

336 correlated to methylation when this gene was up-regulated in cancer while at least one hypo-methylated probe was annotated to this gene or down-regulated in cancer while at least one hyper-methylated probe was annotated to this gene.

We observed no consistent trend when comparing the percentage of hypo-/hypermethylation correlated genes between bidirectional and control genes among TCGA datasets (Fig. 5A, 5B, S4A and S4B). We also compared the percentage of hypo- or hyper-methylated bidirectional genes to the same percentage in random genes and witnessed no consistent trend (Fig. S4C and S4D).

Then we explored the combination of hyper-/hypo-methylation correlated genes in a pair in each TCGA dataset (Fig. 5C, 5D and S4E). The proportion of bidirectional gene pairs comprising two hypo- plus bidirectional gene pairs comprising two hyper-methylation correlated genes was significantly higher than the CG2 except for the BLCA dataset and than the random gene pairs in all the TCGA datasets ( $p$-value $\leq 0.05$, "chisq.test" in R). We didn `t compared the percentage of combination of hyper-/hypo-methylation correlated genes in bidirectional and control gene pairs, since the number of combination of hyper/hypo-methylation correlated genes in CG1 was limited ( $<5$ in each combination in most of TCGA datasets) which could not produce meaningful significance.

In summary, bidirectional genes in a pair were significantly prone to be both hypo- or hyper-methylation correlated genes when comparing with control and random gene pairs despite of percentage of hypo-/hyper-methylation correlated bidirectional genes showing no consistently different from control and random genes.

\section{Correlation between prognosis and bidirectional gene pairs in TCGA datasets}

In previous study, it was indicated that bidirectional gene pairs may play potential role in participating in the development of cancer (Yang et al. 2007). Previous study also showed that certain transcriptional factor can bind and repress the transcription of 
337

338

339

340

341

342

343

344

345

346

347

348

349

350

351

352

353

354

355

356

357

358

359

360

361

362

363

BRCA1 from its bidirectional promoter in cancer (Di et al. 2010). In order to explore the potential clinical outcome of bidirectional genes, we identified prognostic bidirectional and control genes and gene pairs by survival analysis for three endpoints (Overall Survival (OS), disease-free interval (DFI) and progression-free interval (PFI)). We marked genes or gene pairs with Logrank $p$-value $\leq 0.05$ as prognostic genes or gene pairs. The results of survival analysis for all three endpoints in $B G$ and CG1/2 can be viewed in Supplemental file 7, 8 and 9.

Since the protein coding genes are the major function executor compared to other gene types in cells. We firstly evaluated the percentage of prognostic bidirectional genes from protein coding genes. However, there was no consistent trend that the percentage of prognostic protein coding genes in bidirectional genes were significantly higher or lower than control genes in survival analysis for OS, DFI and PFI (Supplemental Table 2). There were also no significantly and consistently different from bidirectional and control genes from all human genes in percentage of prognostic genes in survival analysis for OS, DFI and PFI (Supplemental Table 3).

Then we checked the distribution of prognostic bidirectional gene pairs in 13 TCGA datasets (Supplemental file 10,11 and 12). Briefly, there were no significantly different between bidirectional and control gene pairs in percentage of prognostic gene pairs for survival analysis for all three time endpoints in most of TCGA datasets. (Supplemental Table 4 and 5) and no consistent trend in both gene pairs comprising two protein coding genes and all genes. However, we noticed that the percentage of prognostic bidirectional genes/pairs in LIHC dataset was significantly and consistently higher than the corresponding percentage in control genes/pairs in all analysis except for the gene pairs in survival analysis for DFI.

Remarkably, we found that combination of two genes with different expression level correlated with different survival prognosis in bidirectional and control gene pairs among prognostic gene pairs. The percentage of these bidirectional gene pairs comprising two 
364

365

366

367

368

369

370

371

372

373

374

375

376

377

378

379

380

381

382

383

protein coding genes were significantly higher than control gene pairs (CG1 and CG2) in COAD dataset and significantly lower in none of 13 TCGA datasets in survival analysis for OS (Fig. 6A, 6B and Supplemental file 13).

For example, the two bidirectional genes ENSG00000040531.13 and

ENSG00000262304.1 forming a gene pair, were both prognostic genes in survival analysis for OS, and the down-regulation of both genes may shorten the survival time in HNSC dataset (Fig. 6C and 6D). Moreover low expression of both genes was associated with the poorest prognosis compared with high expression of one gene and high expression of both genes which was associated with the best prognosis in survival analysis for OS (Fig. 6E).

\section{Discussion}

By analyzing the gene expression profiles in TCGA datasets, we identified the up- and down-regulated bidirectional genes and gene pairs. It provided an overview of expression pattern of bidirectional genes and shed a light on the function of bidirectional genes in cancer. In previous study, it indicated that the genes in a bidirectional gene pair were prone to be positively co-expressed (Trinklein et al. 2004). The underlying mechanism was considered to be controlled by a common promoter (bidirectional promoter) and be the co-regulation of two genes in a pair. Our results indicated that the percentage of up-/down-regulated bidirectional genes showed no significant difference from the control and random genes in most of TCGA datasets. However, the percentage of bidirectional gene pairs comprising two up-regulated genes plus gene pairs comprising two down-regulated genes was significant higher than the control and random gene pairs in almost of all TCGA datasets. Bidirectional promoter and coregulation of two genes in a pair may contribute to this phenomenon. It also indicated that the emphasis on the regulation of bidirectional genes lied on the regulation in the pair manner. Besides, a small portion of bidirectional gene pairs comprised one up- 
390

391

392

393

394

395

396

397

398

399

400

401

402

403

404

405

406

407

408

409

410

411

412

413

414

415

regulated gene and one down-regulated gene which was consistent with co-expression analysis from previous study (Trinklein et al. 2004). Although it can be explained by post-transcription process such as regulation by microRNA or different regulation mechanisms on transcription level underlying two genes in a pair. Further studies should be needed to clarify these hypotheses.

We also identified the methylation correlated bidirectional genes. Identification of these methylation correlated genes will be useful for further study in regulation mechanism of bidirectional gene pairs in different cancer types. Like the differentially expressed gene pairs, the bidirectional gene pairs were also prone to be both hypo- or hyper-methylation correlated genes when comparing to the random gene pairs. It suggested that differential methylation may contribute to the expression of bidirectional genes. Again, the emphasis on the regulation of methylation annotated to the bidirectional genes was in the pair manner.

Finally, we found that combination of two genes in a pair with different expression level correlated with different survival outcome for OS. Although the percentage of these gene pairs in bidirectional gene pair were significantly higher than in control gene pair in only one TCGA datasets. It still may indicate functional overlapping of two genes in a bidirectional gene pair.

\section{Conclusions}

In this study, we found that the bidirectional genes in a pair were prone to be both up/down-regulated and regulated by both hypo-/hyper-methylation. Our results emphasized the unique role of expression and regulation of bidirectional genes in pair manner and the functional consequences of both genes in a pair should be considered in the future study.

Acknowledgements: We acknowledge the contributions of the TCGA Research Network. Without their efforts this type of analysis would not be possible. 
416

417

418

419

420

421

422

423

424

425

426

427

428

429

430

431

432

433

434

435

436

437

438

439

440

441

442

443

444

445

446

447

448

449

450

451

452

453

454

455

\section{Reference}

Adachi N, and Lieber MR. 2002. Bidirectional gene organization: a common architectural feature of the human genome. Cell 109:807-809.

Anaya J, Reon B, Chen WM, Bekiranov S, and Dutta A. 2015. A pan-cancer analysis of prognostic genes. PeerJ 3:e1499. 10.7717/peerj.1499

Aran D, Camarda R, Odegaard J, Paik H, Oskotsky B, Krings G, Goga A, Sirota M, and Butte AJ. 2017. Comprehensive analysis of normal adjacent to tumor transcriptomes. Nat Commun 8:1077. 10.1038/s41467-017-01027-z

Cancer Genome Atlas N. 2012a. Comprehensive molecular characterization of human colon and rectal cancer. Nature 487:330-337. 10.1038/nature11252

Cancer Genome Atlas N. 2012b. Comprehensive molecular portraits of human breast tumours. Nature 490:61-70. $10.1038 /$ nature11412

Cancer Genome Atlas Research N. 2012. Comprehensive genomic characterization of squamous cell lung cancers. Nature 489:519-525. 10.1038/nature11404

Cancer Genome Atlas Research N. 2014a. Comprehensive molecular characterization of urothelial bladder carcinoma. Nature 507:315-322. 10.1038/nature12965

Cancer Genome Atlas Research N. 2014b. Comprehensive molecular profiling of lung adenocarcinoma. Nature 511:543-550. 10.1038/nature13385

Cancer Genome Atlas Research N, Weinstein JN, Collisson EA, Mills GB, Shaw KR, Ozenberger BA, Ellrott K, Shmulevich I, Sander C, and Stuart JM. 2013. The Cancer Genome Atlas Pan-Cancer analysis project. Nat Genet 45:1113-1120. 10.1038/ng.2764

Dennis G, Jr., Sherman BT, Hosack DA, Yang J, Gao W, Lane HC, and Lempicki RA. 2003. DAVID: Database for Annotation, Visualization, and Integrated Discovery. Genome Biol 4:P3.

Di LJ, Fernandez AG, De Siervi A, Longo DL, and Gardner K. 2010. Transcriptional regulation of BRCA1 expression by a metabolic switch. Nat Struct Mol Biol 17:1406-1413. 10.1038/nsmb.1941

Hanahan D, and Weinberg RA. 2011. Hallmarks of cancer: the next generation. Cell 144:646-674. 10.1016/j.cell.2011.02.013

Jangid RK, Kelkar A, Muley VY, and Galande S. 2018. Bidirectional promoters exhibit characteristic chromatin modification signature associated with transcription elongation in both sense and antisense directions. BMC Genomics 19:313. 10.1186/s12864-018-4697-7

Koyanagi KO, Hagiwara M, Itoh T, Gojobori T, and Imanishi T. 2005. Comparative genomics of bidirectional gene pairs and its implications for the evolution of a transcriptional regulation system. Gene 353:169-176. 10.1016/j.gene.2005.04.027

Lee TI, and Young RA. 2013. Transcriptional regulation and its misregulation in disease. Cell 152:1237-1251. 10.1016/j.cell.2013.02.014

Lin JM, Collins PJ, Trinklein ND, Fu Y, Xi H, Myers RM, and Weng Z. 2007. Transcription factor binding and modified histones in human bidirectional promoters. Genome Res 17:818-827. 10.1101/gr.5623407

Liu B, Chen J, and Shen B. 2011. Genome-wide analysis of the transcription factor binding preference of human bidirectional promoters and functional annotation of related gene pairs. BMC Syst Biol 5 Suppl 1:S2. 10.1186/1752-0509-5-S1-S2

Liu J, Lichtenberg T, Hoadley KA, Poisson LM, Lazar AJ, Cherniack AD, Kovatich AJ, Benz CC, Levine DA, Lee AV, 
456

457

458

459

460

461

462

463

464

465

466

467

468

469

470

471

472

473

474

475

476

477

482
Omberg L, Wolf DM, Shriver CD, Thorsson V, Cancer Genome Atlas Research N, and Hu H. 2018. An Integrated TCGA Pan-Cancer Clinical Data Resource to Drive High-Quality Survival Outcome Analytics. Cell 173:400-416 e411. 10.1016/j.cell.2018.02.052

Morris TJ, Butcher LM, Feber A, Teschendorff AE, Chakravarthy AR, Wojdacz TK, and Beck S. 2014. ChAMP: 450k Chip Analysis Methylation Pipeline. Bioinformatics 30:428-430. 10.1093/bioinformatics/btt684

Risso D, Ngai J, Speed TP, and Dudoit S. 2014. Normalization of RNA-seq data using factor analysis of control genes or samples. Nat Biotechnol 32:896-902. 10.1038/nbt.2931

Robinson MD, McCarthy DJ, and Smyth GK. 2010. edgeR: a Bioconductor package for differential expression analysis of digital gene expression data. Bioinformatics 26:139-140. 10.1093/bioinformatics/btp616

Shu J, Jelinek J, Chang H, Shen L, Qin T, Chung W, Oki Y, and Issa JP. 2006. Silencing of bidirectional promoters by DNA methylation in tumorigenesis. Cancer Res 66:5077-5084. 10.1158/0008-5472.CAN-05-2629

Tang CS, and Epstein RJ. 2007. A structural split in the human genome. PLoS One 2:e603. 10.1371/journal.pone.0000603

Thompson JA, Christensen BC, and Marsit CJ. 2018. Pan-Cancer Analysis Reveals Differential Susceptibility of Bidirectional Gene Promoters to DNA Methylation, Somatic Mutations, and Copy Number Alterations. Int J Mol Sci 19. 10.3390/ijms19082296

Trinklein ND, Aldred SF, Hartman SJ, Schroeder DI, Otillar RP, and Myers RM. 2004. An abundance of bidirectional promoters in the human genome. Genome Res 14:62-66. 10.1101/gr.1982804

Xiao B, Chen L, Ke Y, Hang J, Cao L, Zhang R, Zhang W, Liao Y, Gao Y, Chen J, Li L, Hao W, Sun Z, and Li L. 2018. Identification of methylation sites and signature genes with prognostic value for luminal breast cancer. BMC Cancer 18:405. 10.1186/s12885-018-4314-9

Yang M, and Elnitski L. 2014. Orthology-driven mapping of bidirectional promoters in human and mouse genomes. BMC Bioinformatics 15 Suppl 17:S1. 10.1186/1471-2105-15-S17-S1

Yang MQ, Koehly LM, and Elnitski LL. 2007. Comprehensive annotation of bidirectional promoters identifies coregulation among breast and ovarian cancer genes. PLoS Comput Biol 3:e72.

10.1371/journal.pcbi.0030072 


\section{Figure 1 (on next page)}

\section{Identification of bidirectional gene pairs in the human genome}

A. Schematic representation of bidirectional gene pair (BG), control gene pair (CG1 and CG2) and random gen pair organization. TSS, transcription start site. Arrow on gene indicating the transcriptional orientation.

B. Distribution of gene types in bidirectional and control genes (CG1).

C. Distribution of the combinations of gene types in bidirectional and control gene pairs (CG1).

D. Gene ontology (GO) showing the function enrichment of bidirectional genes. Red dash line indicate the significant cutoff. 
A

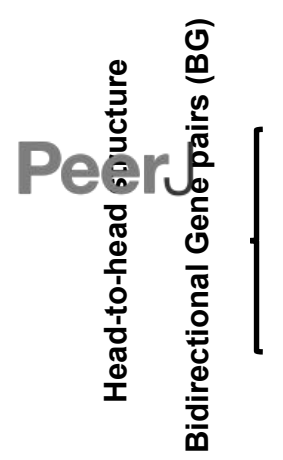<smiles>[H]/C=C/C=C1CCCC1</smiles>

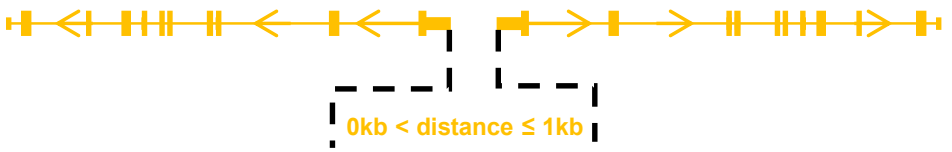

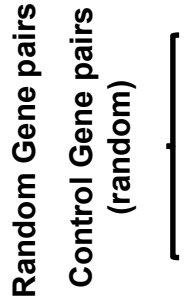

B

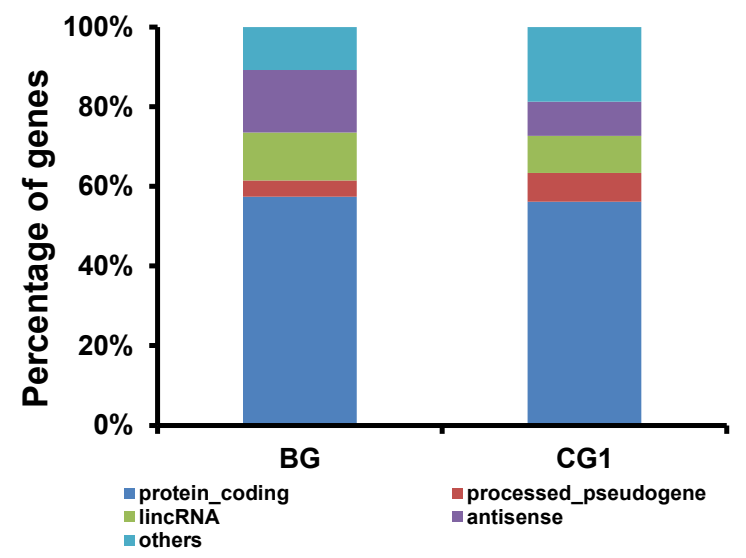

Gene1 randomly chosen from genome

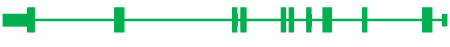

Gene2 randomly chosen from genome

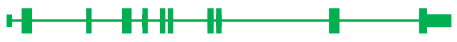

C

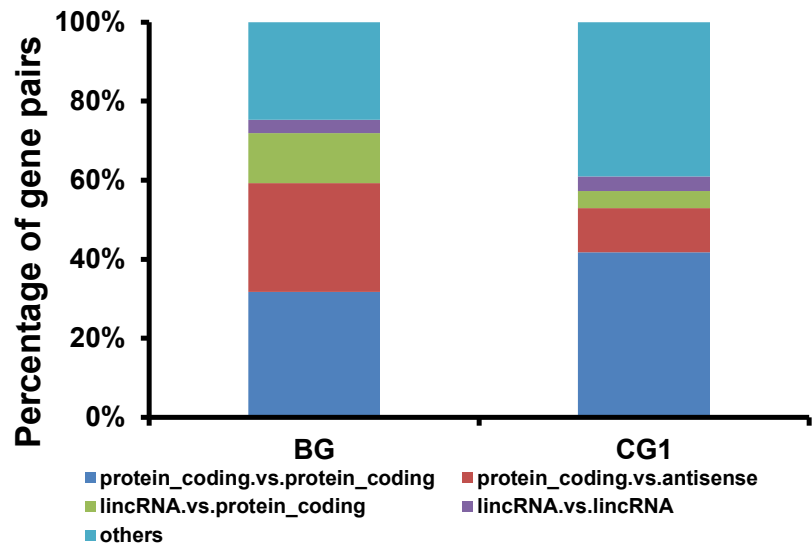

GO:0032212 positive regulation of telomere maintenance via telomerase GO:0031297 replication fork processing GO:0000724 double-strand break repair via homologous recombination

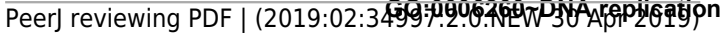
GO:0007067 mitotic nuclear division GO:0051301 cell division GO:0006281 DNA repair

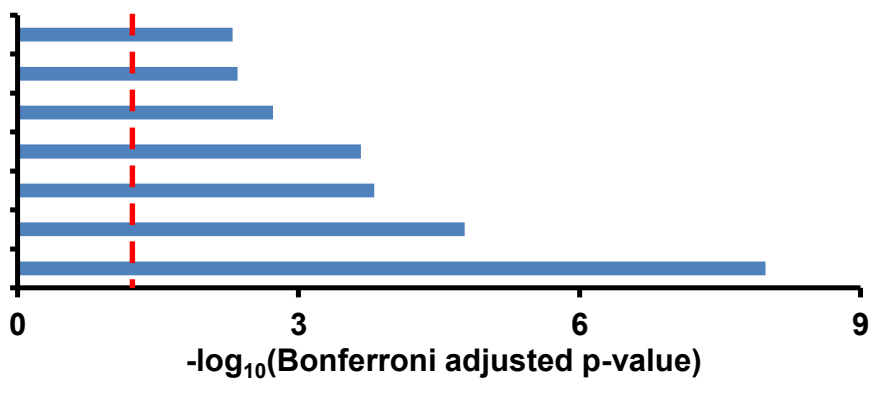




\section{Figure 2 (on next page)}

\section{Identification and characterization of differentially expressed bidirectional genes in TCGA dataset}

A. Percentage of expressed and non-expressed bidirectional genes among TCGA datasets. X-axis, TCGA datasets.

B. Percentage of expressed and non-expressed control genes (CG1) among TCGA datasets. X-axis, TCGA datasets.

C. Percentage of up-regulated bidirectional and control genes (CG1) among TCGA datasets. X-axis, TCGA datasets. The p-values were computed by "chisq.test" function in R. "ns" indicating not significant. "*" indicating $p$-value $<0.05$. "**" indicating $p$-value $<0.01$. " $* * * "$ indicating $p$-value $<0.001$.

D. Percentage of down-regulated bidirectional and control genes (CG1) among TCGA datasets. X-axis, TCGA datasets. The p-values were computed by "chisq.test" function in R. "ns" indicating not significant. "*" indicating $p$-value $<0.05$. "**" indicating $p$-value $<0.01$. " $* * * "$ indicating $p$-value $<0.001$. 
A

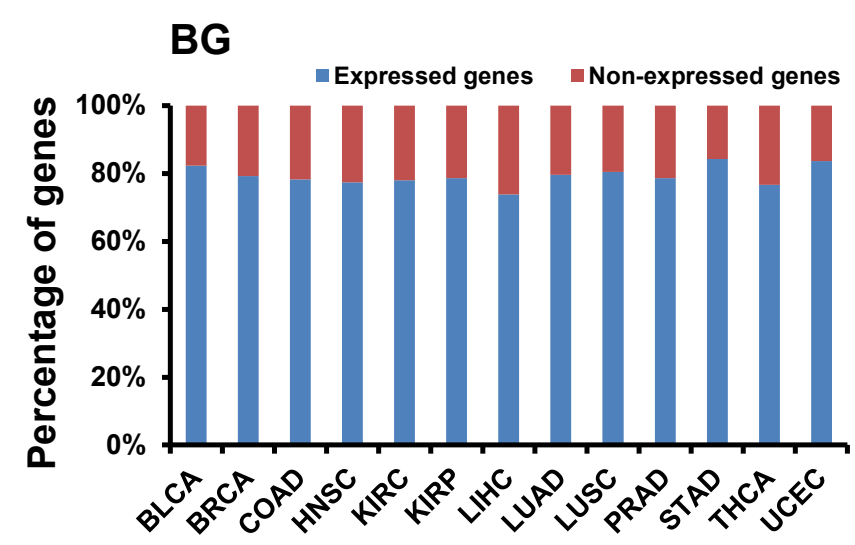

C

Up-regulation

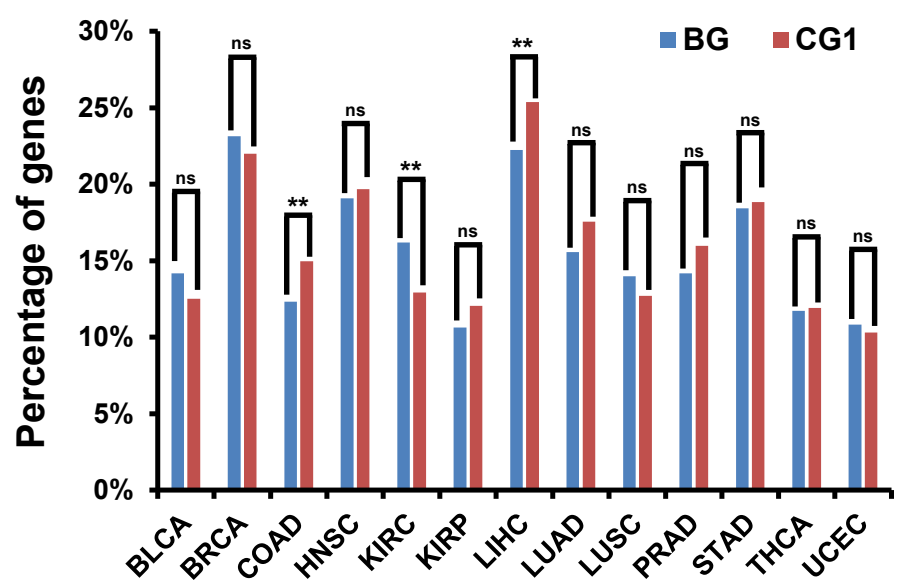

B

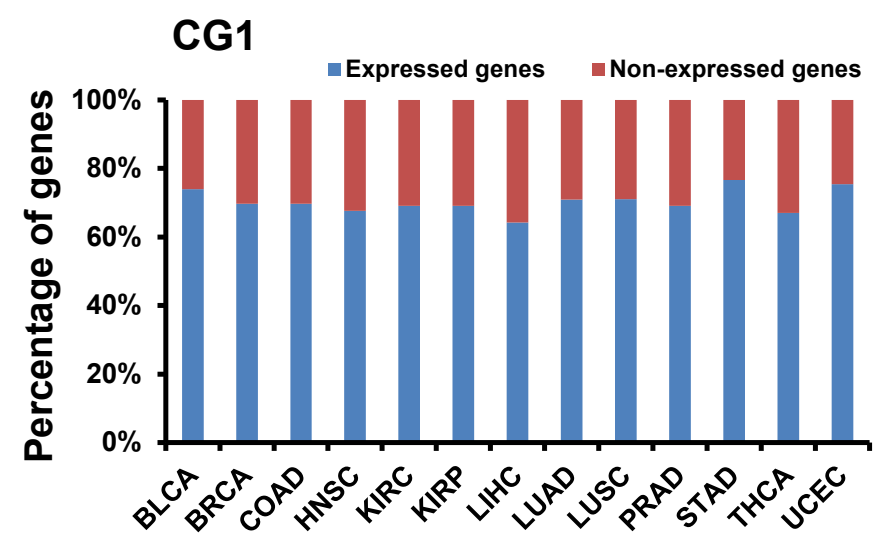

D

Down-regulation

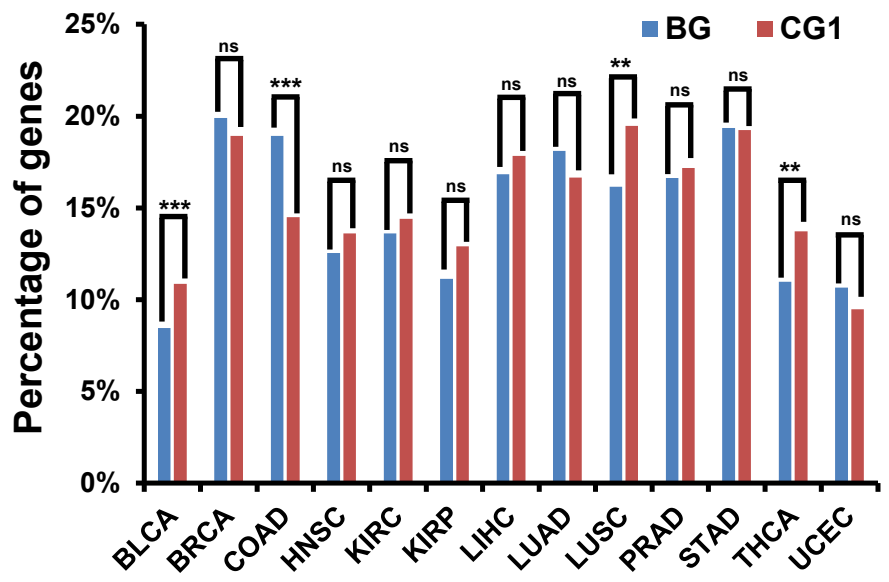


Figure 3 (on next page)

Identification and characterization of differentially expressed bidirectional gene pairs in TCGA dataset

A. Percentage of expressed and non-expressed bidirectional gene pairs among different TCGA datasets. Xaxis, TCGA datasets.

B. Percentage of expressed and non-expressed control gene pairs (CG1) among different TCGA datasets. Xaxis, TCGA datasets.

C. Percentage of the patterns of differentially expressed bidirectional combination in a pair. X-axis, TCGA datasets.

D. Percentage of the patterns of differentially expressed control combination in a pair (CG1). X-axis, TCGA datasets. 


\section{PeerJ}

A

BG

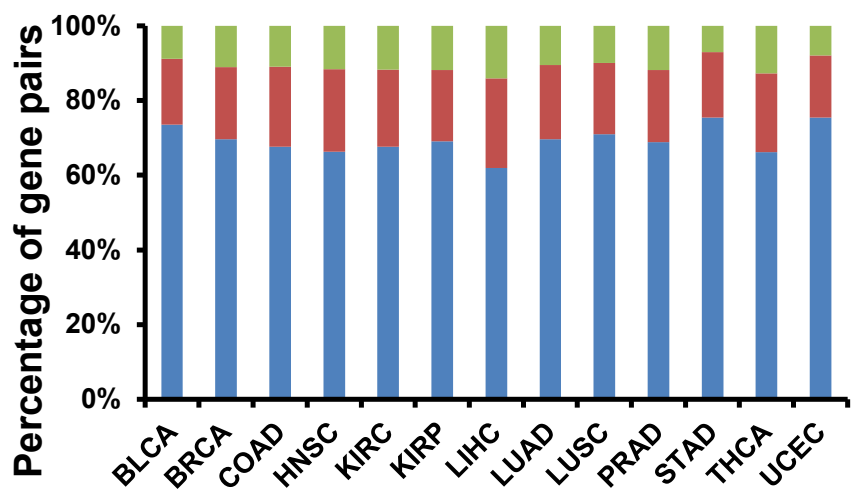

nonexpressed_vs_nonexpressed Expressed_vs_nonexpressed

- Expressed_vs_expressed

C

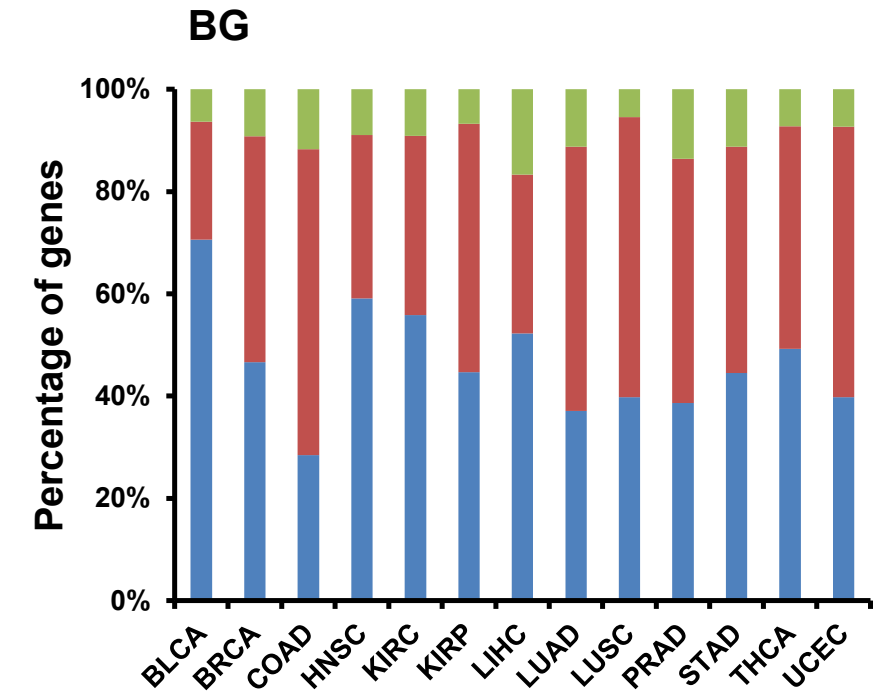

up_vs_up nown_vs_down

up_vs_down
B

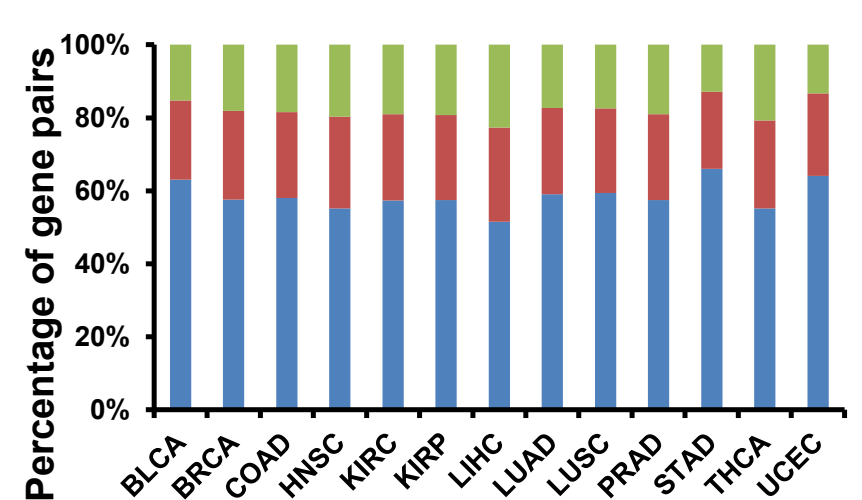

Nonexpressed_vs_nonexpressed Expressed_vs_nonexpressed

Expressed_vs_expressed

D

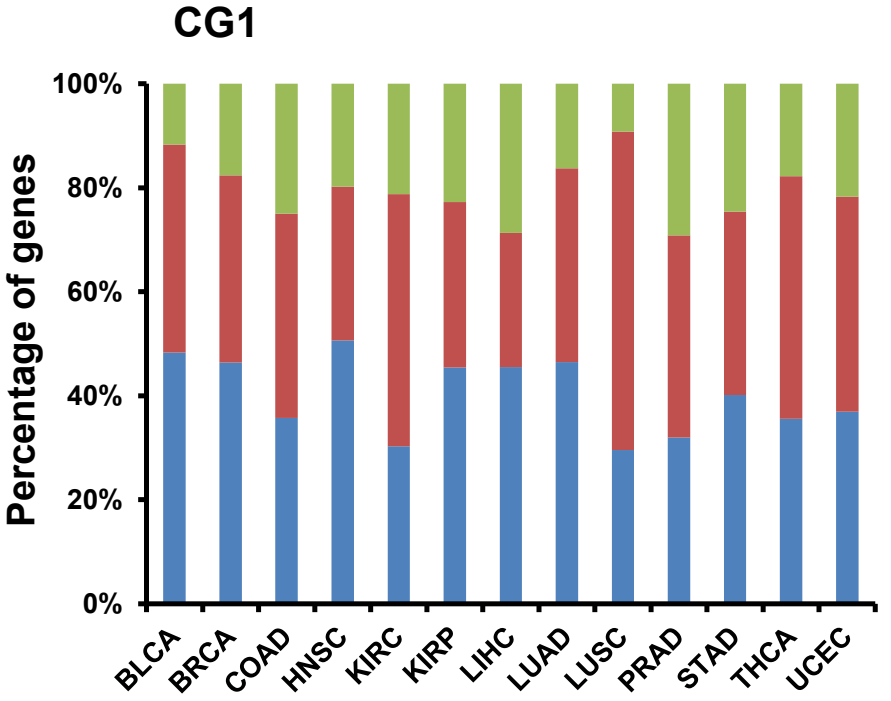

up_vs_up

n down_vs_down

up_vs_down 


\section{Figure $\mathbf{4}$ (on next page)}

\section{Characterization of up-/down-regulated bidirectional genes and gene pairs in multiple} TCGA datasets

A. Percentage of bidirectional, control and random genes up-regulated in a specific number of TCGA datasets. Each number " $n$ " on the $x$-axis indicates the corresponding percentage of up-regulated genes on the $y$-axis in " $n$ " of 13 TCGA datasets. The percentages in random genes represent mean of 100 random sets and the error bars represent the standard deviation. The p-values were computed by "chisq.test" function in R. The number of up- or down-regulated random genes in a specific number of TCGA datasets used in "chisq.test" is the mean of 100 random sets.

B. Percentage of bidirectional, control and random genes down-regulated in a specific number of TCGA datasets. Each number " $n$ " on the $x$-axis indicates the corresponding percentage of down-regulated genes on the $y$-axis in " $n$ " of 13 TCGA datasets. The percentages in random genes represent mean of 100 random sets and the error bars represent the standard deviation. The p-values were computed by "chisq.test" function in R. The number of up- or down-regulated random genes in a specific number of TCGA datasets used in "chisq.test" is the mean of 100 random sets.

C. Percentage of bidirectional, control and random gene pairs with specific combination of up- or downregulated genes in specific number of TCGA datasets. Each number " $n$ " on the $x$-axis means corresponding percentage on the $y$-axis of the specific combinations of up- or down-regulated gene pairs in " $n$ " of 13 TCGA datasets. The percentages in random genes represent mean of 100 random sets and the error bars represent the standard deviation. The p-values were computed by "chisq.test" function in $\mathrm{R}$. The number of specific combination in random gene pairs in a specific number of TCGA datasets used in "chisq.test" is the mean of 100 random sets.

D. Percentage of bidirectional, control and random gene pairs with specific combination of up- or downregulated genes in specific number of TCGA datasets. Each number " $n$ " on the $x$-axis means corresponding percentage on the $y$-axis of the specific combinations of up- or down-regulated gene pairs in " $n$ " of 13 TCGA datasets. The percentages in random genes represent mean of 100 random sets and the error bars represent the standard deviation. The $p$-values were computed by "chisq.test" function in $\mathrm{R}$. The number of specific combination in random gene pairs in a specific number of TCGA datasets used in "chisq.test" is the mean of 100 random sets. 


\section{PeerJ}

A

Up-regulation

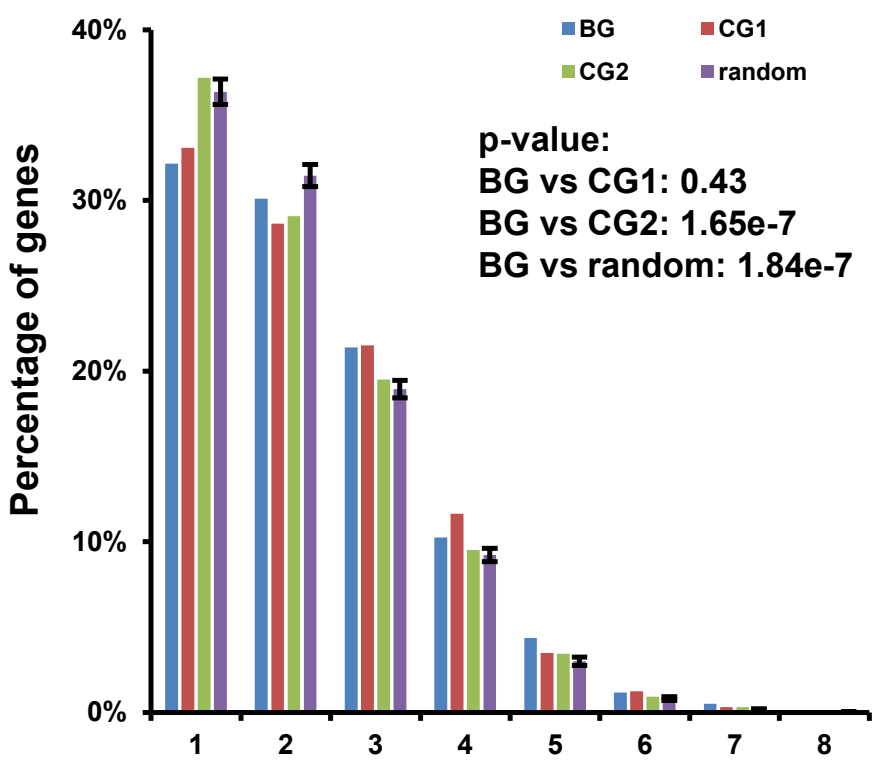

C

up_vs_up + down_vs_down

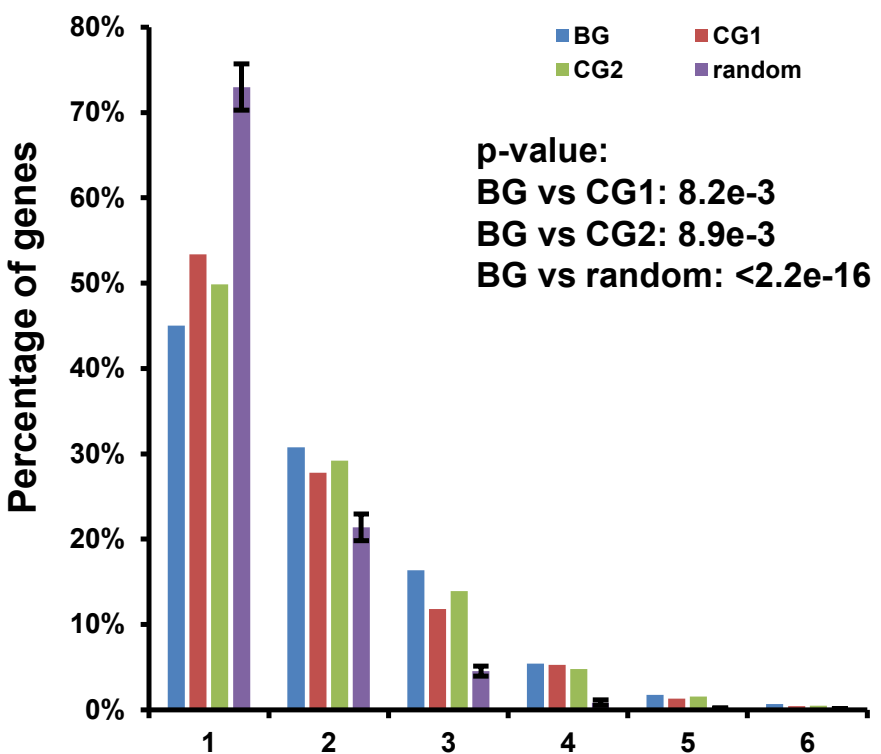

\section{Manuscript to be reviewed}

B

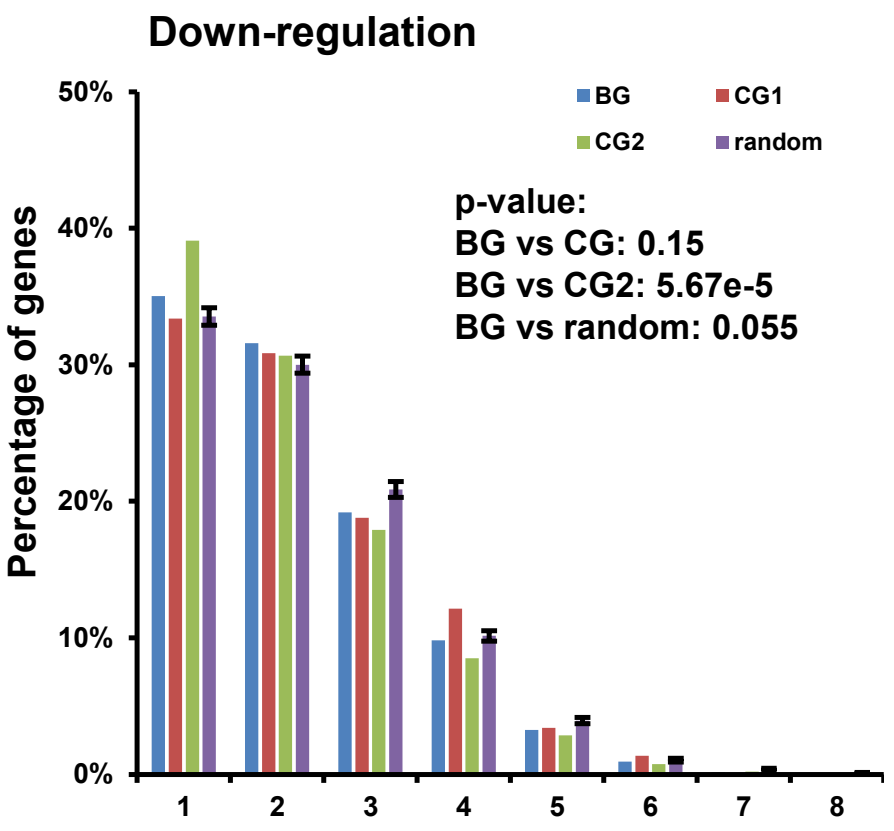

D

up_vs_down

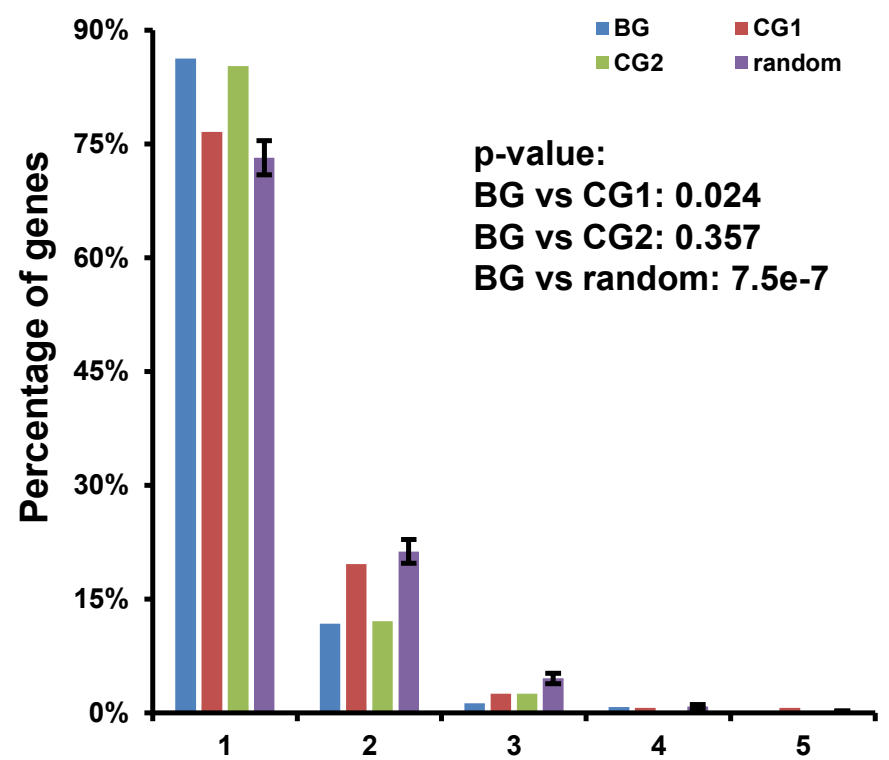




\section{Figure 5 (on next page)}

Correlation of methylation levels and bidirectional genes

A. Percentage of hypo-methylation correlated bidirectional and control genes (CG1) in TCGA dataset. X-axis, TCGA datasets. The p-values were computed by "chisq.test" function in R. "ns" indicating not significant. "*" indicating $p$-value $<0.05$. "**" indicating $p$-value $<0.01$. "***" indicating $p$-value $<0.001$.

B. Percentage of hyper-methylation correlated bidirectional and control genes (CG1) in TCGA dataset. Xaxis, TCGA datasets. The p-values were computed by "chisq.test" function in R. "ns" indicating not significant. " $*$ " indicating $p$-value $<0.05$. " $* *$ " indicating $p$-value $<0.01$. " $* * *$ " indicating $p$-value $<0.001$.

C. Percentage of combination of hypo- or hyper- methylation correlated bidirectional genes in TCGA dataset. $\mathrm{X}$-axis, TCGA datasets.

D. Percentage of combination of hypo- or hyper- methylation correlated control genes (CG2) in TCGA dataset. X-axis, TCGA datasets. 
PeerJ

$\mathbf{A}$

Hypo-methylation correlated genes

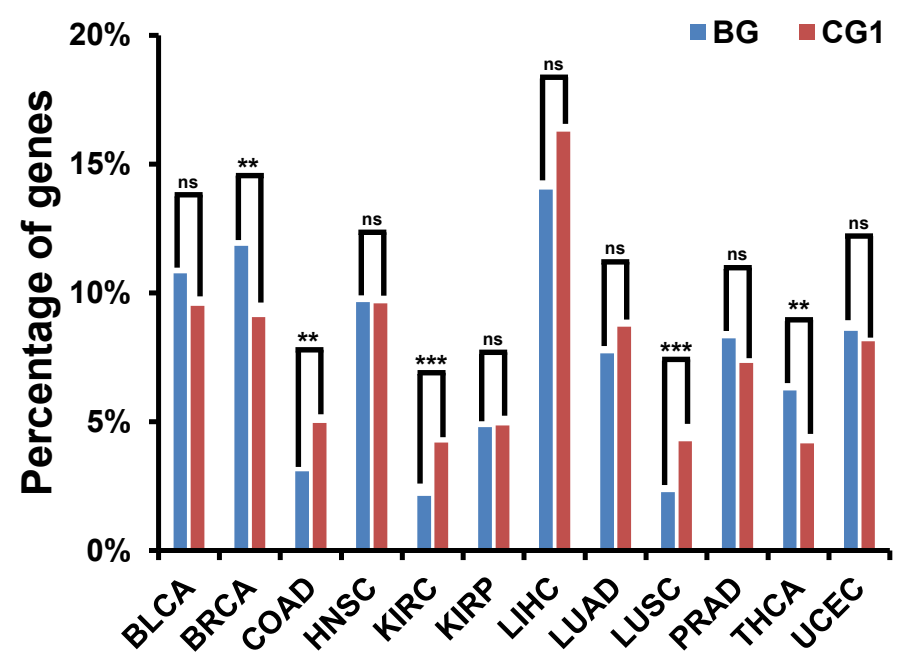

C

BG

.

.

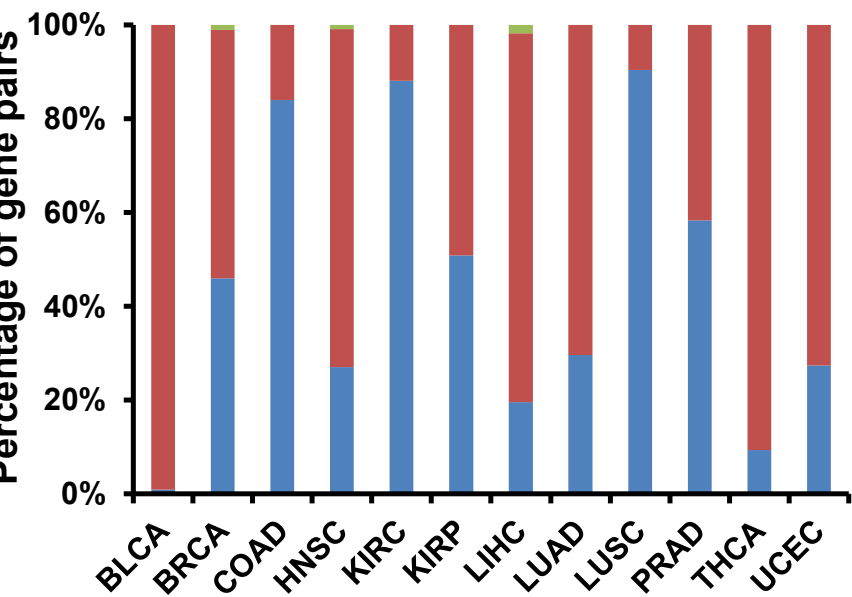

-Hyper_vs_Hyper $\quad$ Hypo_vs_Hypo

-Hyper_vs_Hypo

\section{Manuscript to be reviewed}

B

Hyper-methylation correlated genes

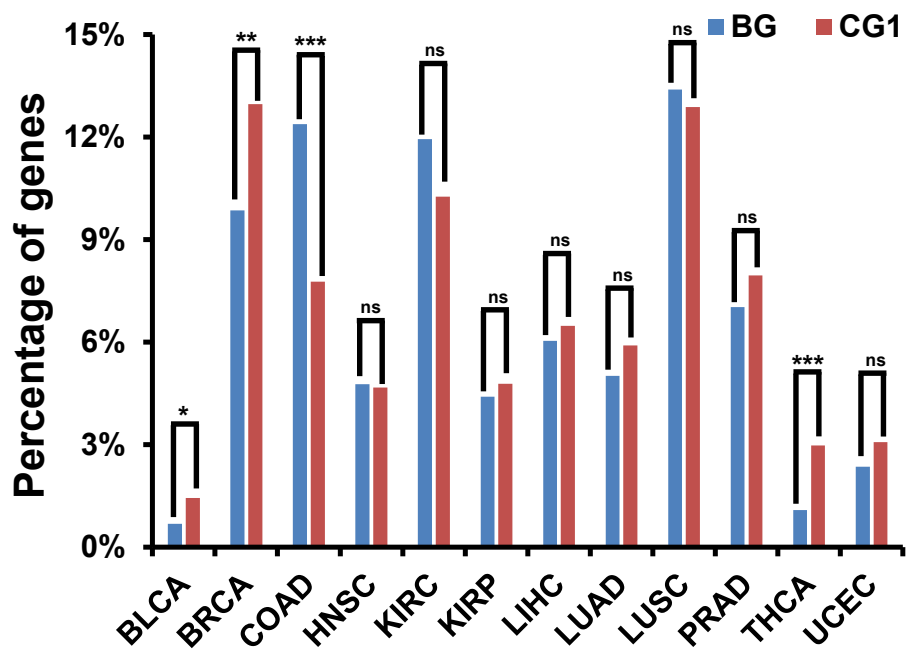

D

CG2

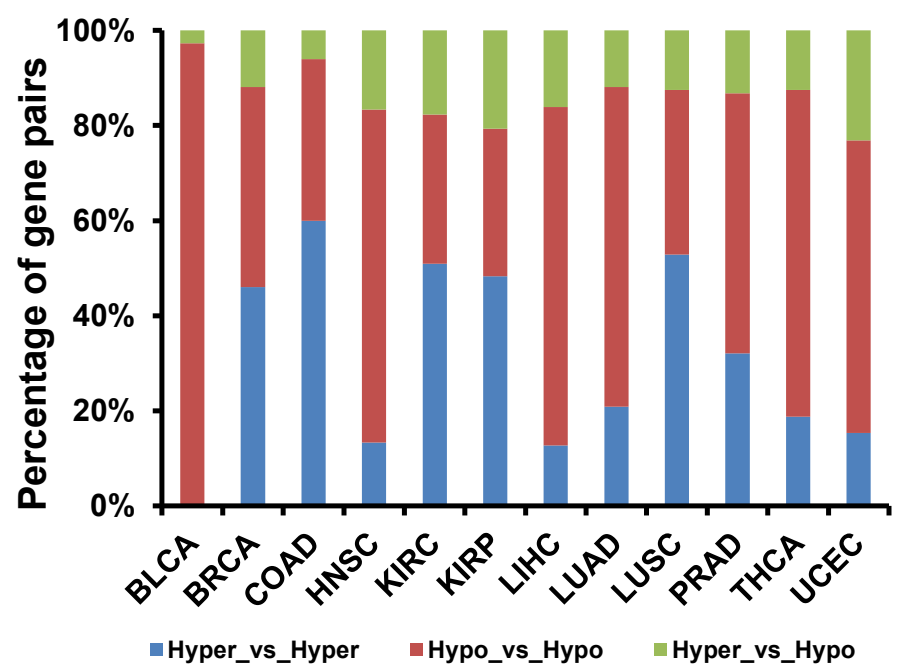




\section{Figure 6 (on next page)}

\section{Correlation of survival rate and bidirectional genes or gene pairs in TCGA datasets}

A. Percentage of candidate prognostic gene pairs comprising two protein coding genes among all prognostic bidirectional and control gene pairs (CG1) comprising two protein coding genes in survival analysis for OS. Candidate prognostic gene pair were identified as two genes in this pair with different expression level correlated with different survival outcome. The p-values were computed by "chisq.test" function in R. "ns" indicating not significant. "*" indicating $p$-value $<0.05$. "**" indicating p-value $<0.01$. " ***" indicating $p$ value $<0.001$.

B. Percentage of candidate prognostic gene pairs comprising two protein coding genes among all prognostic bidirectional and control gene pairs (CG2) comprising two protein coding genes in survival analysis for OS. Candidate prognostic gene pair were identified as two genes in this pair with different expression level correlated with different survival outcome. The p-values were computed by "chisq.test" function in R. "ns" indicating not significant. "*" indicating p-value $<0.05$. "**" indicating $\mathrm{p}$-value $<0.01$. " $* * *$ " indicating $\mathrm{p}$ value $<0.001$

C. Survival plot for bidirectional genes, ENSG00000040531.13. Median value of TMM normalized expression value was used as cutoff to divide samples into four groups.

D. Survival plot for bidirectional genes, ENSG00000262304.1. Median value of TMM normalized expression value was used as cutoff to divide samples into four groups.

E. Survival plot for bidirectional gene pair. Median value of TMM normalized expression value was used as cutoff to divide samples into four groups. 
A

B

protein_coding gene vs protein_coding gene

protein_coding gene vs protein_coding gene
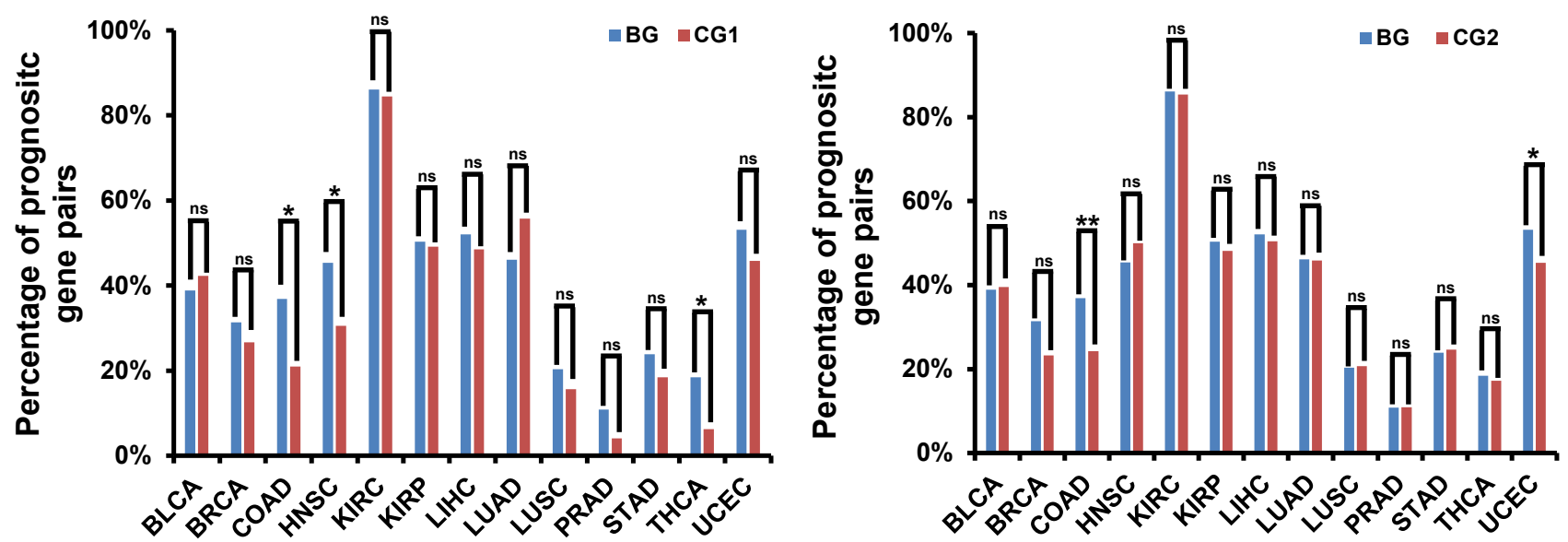

C

D

E

ENSG00000040531.13

ENSG00000262304.1
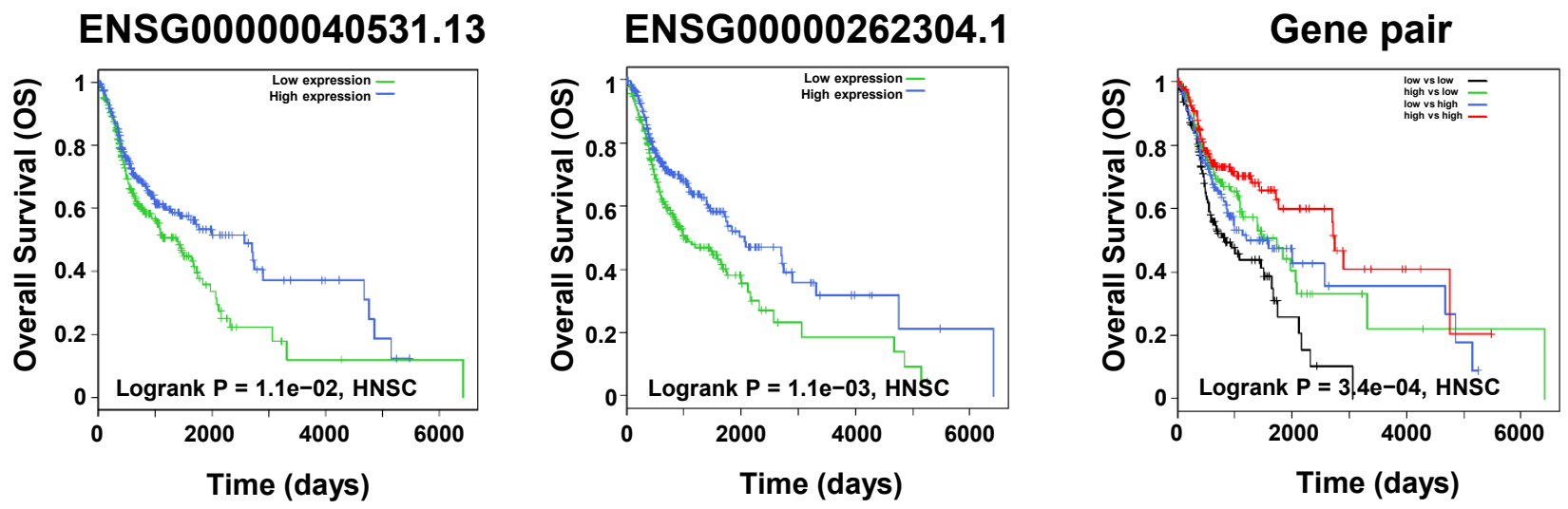